\title{
Addressing the health care needs of people who identify as transgender: What do nurses need to know?
}

Ilana Boucher, Sharon L. Bourke, Janet Green, Elianna Johnson, Linda K. Jones

School of Nursing and Healthcare Professions, Melbourne, Australia

Received: January 23, 2020

DOI: $10.5430 /$ ijh.v6n2p14
Accepted: April 20, 2020

Online Published: April 30, 2020

\begin{abstract}
Being transgender (TG) is part of the natural spectrum of human diversity, and its visibility has increased with societal change. The TG population is comprised of individuals identifying themselves as the opposite gender to that which they were born. Adult TG people are calculated as a part of Australia's non-heterosexual marginal population; therefore, exact numbers are unknown. As a result of not being recognised, TG people have faced multiple challenges, fairing worse in all socioeconomic and health measures, including not being able to afford and access appropriate healthcare. Many of these challenges arise from a lack of understanding resulting in social exclusion, bullying, and physical attacks. The isolation and physical assaults on this community creates anxiety and mental health conditions, including self-harm, suicide, depression, personality disorder, psychosis, post traumatic disorder, and eating disorders. To affirm a gender identity that is different from the gender a person was born to is referred to as "transition." The lengths to which an individual will go to transition is varied with some people choosing to change only their dress and mannerisms and others to undertake medical interventions such as hormonal therapy and/or surgery. In Australia, there is limited capacity within the health system to support the needs of the trans and gender diverse (TGD) population. Initiatives such as nurse-led post-operative support service need to be created as a way to address this. This service could provide patients with nurse advocates working collaboratively with other health professionals to provide primary health solutions.
\end{abstract}

Key Words: Transgender, Gender identity, Gender dysphoria, Nursing, Mental health, Surgery, Cultural safety

\section{INTRODUCTION}

Being transgender (TG) or gender diverse (GD) (TGD) is part of the natural spectrum of human diversity, and its visibility has increased with the social changes occurring in the broader lesbian, gay, bisexual, TG, questioning, intersex (LBTQI+) community and society as a whole. It is noteworthy that there is an important difference between sex and gender. Gender is an internal sense of being male or female and is based on societal or cultural definitions of masculine or feminine. Sex refers to differences in chromosomes, hormones and external and internal sex organs. ${ }^{[1]}$ The TG population is comprised of individuals identifying themselves as the opposite gender to that which they were born, termed gender dysphoria (GDy). ${ }^{[2]}$ As a result of this, some TG people may transition to the sex that they identify with the most. This transition may involve hormone or gender affirmation surgeries or a combination of both. ${ }^{[3]}$ With increasing visibility and social acceptance of gender diversity in countries such as Australia, more TGD children and adolescents are presenting to community health services and specialist medical services. ${ }^{[4]}$

*Correspondence: Sharon L. Bourke; Email: s.bourke@ @ederation.edu.au; Address: PO Box 859, Berwick, VIC 3806, Australia. 
This paper is timely and important because it has been estimated that about $1.2 \%$ of Australian adolescents identify as TG, therefore referrals to health care professionals will continue to rise and nurses need to be aware of the issues impacting the TGD population. ${ }^{[4]}$

\subsection{Methodology}

An integrative review methodology was utilised here as it enables a broad review and facilitates a comprehensive understanding of the phenomenon. The literature was gathered using a framework outlined as Arksey and O'Malley's 5 step method. ${ }^{[5]}$ Relevant and recent literature was identified using Boolean phrase terms such as TG, gender identity, mental health, cultural safety. The Cumulative Index of Nursing and Allied Health (CINAHL) Complete, pubMED, Humanities Source were the databases of choice. This search identified 4,600 results. These terms as well as healthcare professional, nurses, staff or professionals or education narrowed the search to 1,220 . Using only full text, peer reviewed journal articles within the last 10 years identified 377 articles of relevance. Finally, with the addition of surgery, surgical, six articles were identified that made a clear link to the needs to the TG population and their healthcare requirements.

\subsection{Australian statistics}

GDy is estimated to occur in approximately 25 million people worldwide. ${ }^{[6]}$ Adult TG people are calculated as a part of Australia's non-heterosexual marginal population; therefore, exact numbers are unknown. The non-heterosexual community was estimated to be $3.2 \%$ in the 2016 census. ${ }^{[2]}$ As a result of not being recognised, TG people have faced multiple challenges, fairing worse in all socioeconomic and health measures, including not being able to afford and access appropriate healthcare. ${ }^{[7]}$ The Australian government and the medical profession place minimal emphasis on researching and supporting the needs of the TG population, leaving health professionals disadvantaged in understanding their medical, holistic and legal needs. ${ }^{[8-10]}$

\subsection{Gender identity}

Gender identity, or the sense of being male or female develops early, with most children approximately 2 years of age being able to identify their own gender, and most identify with the sex they were at birth. ${ }^{[11]}$ GDy is the distress a person feels due to a mismatch between their gender identity and their sex assigned at birth. People who experience GDy are typically TG. ${ }^{[12]}$ The onset of cross-gender interests and activities is usually between ages 2 and 4 years, with many parents later reporting that their child has always had cross-gender interests. ${ }^{[12]}$ The issue is that individuals grap- pling with GD struggle with the development of secondary sex characteristics during adolescence. The causes of GDy are currently unknown, however hormonal influences in the womb, genetics and environmental factors are all suspected to be involved. ${ }^{[11,12]}$ The reported prevalence has been increasing, with most estimates suggesting that as many as 521 in 100,000 males and 265 in 100,000 females experience GDy. ${ }^{[11]}$

\subsection{Historical perspectives}

Transwomen/female (born male) and transmen/male (born female) have been a part of society in every culture at every time. ${ }^{[11]}$ Their frequency and visibility is a function of societal mores, and in most societies they have suffered discrimination or worse. ${ }^{[12]}$ Transgenderism and GDy can be dated back to millennia. Historically in 222 AD Roman Emperor Elagabalus was assassinated at 18 years of age for his sexual and religious behaviour. Emperor Elagabalus was credited as the first head of state to offer a reward to medical practitioners whom could give him female genitalia. ${ }^{[13]}$ Then in 1885 the Criminal Law Act outlawed all homosexual and transgenderism behaviour in the United Kingdom. ${ }^{[14]}$ The historical underpinnings in Australia have been strongly influenced by international law. During the Colonial era; the development of Australian Law was heavily impacted upon United Kingdom rule; leading to the outlawing of transgenderism behaviour in Australia. ${ }^{[14]}$

Moving into the $20^{\text {th }}$ century, the term "transgenderism" was used to describe the unlawful behaviour of TG and transsexual males and females, transvestites, drag queens, and/or any individual that deviates from expected gender society norms. ${ }^{[14]}$ However, mid-20 ${ }^{\text {th }}$ century saw a change in social norms contributing to both the development of both male-female and female-male gender reassignment surgery primarily in both the United States and Europe in the 1950's and use of the term "transgender". [13]

Continual social stigmatisation and unlawful behaviour led to initial TG research to be secretive in nature. ${ }^{[15]}$ Australia's first Gender Dysphoria Clinic opened in Melbourne in 1975 with the first gender affirmation surgery undertaken in 1976. ${ }^{[15]}$ Transgenderism and body dysmorphia, however, were still perceived solely as a psychological issue leading to inequities in health care access for this population. ${ }^{[14]}$ Transgenderism was only recognised as a body dysmorphia and not a psychological disorder in the mid to late 1990's leading to new developments of health services for this population. ${ }^{[14]}$ The TG community was first acknowledged in the 2011 Australian census despite being depicted in Aboriginal drawings centuries ago, being referred to as "Sistergirls" and "Brotherboys" in Aboriginal culture today. ${ }^{[7]}$ 


\section{Challenges associated With being TG}

The TG population faces multiple challenges, many arising from a lack of understanding resulting in social exclusion, bullying, physical attacks, some resulting in murder. Resultant isolation and physical assaults on this community creates anxiety and mental health conditions, with $50 \%$ of young trans-genders partaking in self-harm and $41 \%$ trying to take their own lives compared to $5 \%$ in general population. ${ }^{[2,10]}$ Unique risk factors have contributed to the high suicide rate in the TG population. These include lack of family and social support networks, TG based abuse and violence, GDy and body related shame, being part of another minority and gender based discrimination from both the general population and from health care professionals. ${ }^{[3,16]}$ In addition, these children/adolescents may experience other mental health issues such as depression (75\%), personality disorder (20\%), psychosis (16\%), post traumatic disorder (25\%), and eating disorders $(23 \%) .{ }^{[10]}$

Social isolation and abuse also leads to a higher incidence of tobacco, drug and alcohol abuse within this population, which in turn leads to increased risky behaviour and poorer health outcomes (Comfort \& McCausland, 2013). ${ }^{[17]}$ Furthermore, the likelihood of childhood sexual abuse and rape has increased in this population, including abuse from their partners, increasing the risks of sexual trauma. ${ }^{[7,8]}$ Overall there is discrimination which begins at a young age when the signs of GDy are being displayed at 3 to 4 years of age, placing this population at risk of bullying and discrimination as soon as they commence school. ${ }^{[10]}$ The Royal Children's Hospital Melbourne reports that these children/adolescents may experience severe psychiatric morbidity; with a high incidence of anxiety (72.2\%), personality disorders (20.1\%), psychosis (16.2\%), post-traumatic disorder (25.1\%), and eating disorders (22.7\%), evidencing that this populations health is at a higher risk. ${ }^{[10]}$

Delays for appropriate medical interventions when they wish to transition to their identified gender, leads to undue stress with over $70 \%$ diagnosed with depression. ${ }^{[18]}$ This impacts on their social, educational and work life as they are viewed as not conforming to birth gender norms. ${ }^{[19]}$ Finding safe employment is also a challenge for this population, and could be a contributing factor to the high levels of the TG community working in the sex industry, placing them at higher risk of sexually transmitted diseases (STIs), Human Immunodeficiency (HIV) and anal cancer. ${ }^{[8]}$ Indeed, the World Health Organisation (WHO) stated TG women are approximately 49 times more likely to be living with HIV, and in some countries this rate is 80 times that of the general adult population. ${ }^{[20]}$ Employment limitations give this population a lower socioeconomic standing restricting access to safe, affordable housing. Being displaced by families/communities when voicing their gender identity places this population at higher risk of being homeless from a young age, increasing their risk of being victims of violent crimes and associated health complications. ${ }^{[21]}$ The discrimination and transphobia that exists in society is still present in support agencies who assist the homeless. With a shortage of TG agencies looking out for this population they are at higher risk of being subjected to violent crimes, until the government enforces training in this area and places more emphasis on protecting this population. ${ }^{[21]}$

\subsection{Body dysmorphia in children}

Inequities are faced by TG people when children experiencing GDy cannot access specialist services in their community. ${ }^{[9]}$ Seeking treatment outside of their geographical area can delay hormonal treatment, or create a considerable expense to families, who wish to prevent the development of original features and secondary sexual characteristics. This is distressing to the child and causes him/her undue stress and pain. ${ }^{[22]}$ The expense and inconvenience of travel are further compounded by the fact that minors' families must seek court approval for hormonal treatment at their own expense, once again limiting treatment to all. ${ }^{[9,22]}$ In the past, courts in Australia have determined the TGD adolescent's ability to access hormones and other treatment. Several successful cases in the Family Court of Australia ${ }^{[23]}$ allowed for medical clinicians to determine an adolescents' capacity to provide informed consent for treatment. ${ }^{[24]}$ It is important to note that the clinical needs of children and pre-pubertal adolescents are different.

For those children who can access and afford hormonal treatment, they require ongoing specialist care for the side effects of their treatment. There is a shortage of funding and medical specialists in this area with Victoria providing the most services in Australia. ${ }^{[10]}$ This lack of funding also extends into the research that is related to people who identify as TG. For instance, there is very little research into the health status and life expectancy of this population, placing them at higher risk of experiencing preventable diseases and unnecessary death. ${ }^{[8,25]}$ The TG population has been identified as having a higher incidence of attention deficit disorder and autism, being four times higher than the general population. This further highlights the need to access specialist care within local communities to prevent disadvantaging this population any further. ${ }^{[26]}$ 


\subsection{Seeking gender reassignment}

To affirm a gender identity that is different from the gender a person was born to is referred to as "transition." The lengths to which an individual will go to transition is varied with some people choosing to change only their dress and mannerisms and others to undertake medical interventions such as hormonal therapy and/or surgery. Often referred to as gender affirming or confirming, surgeries can take many forms depending on the transition the person wishes to take and can involve many subspecialties including urologists, gynaecologists, and maxillofacial surgeons. ${ }^{[27]}$

It has been recommended that there be a multidisciplinary approach to gender transition, which includes input from general medical practitioners, psychiatrists, psychologists, endocrinologists, and plastic surgeons. ${ }^{[6]}$ With all of these specialists involved, it is no wonder that some find the costs of transition prohibitively high. The process to transition to the gender identified should start with discussions of fertility preservation, with both trans males and trans females offered the opportunity to store their biological semen and eggs. ${ }^{[24]}$ This would then be followed by the suppression of puberty and the prevention of secondary sex characteristics (breast growth and menstruation in trans males and voice deepening and facial hair development in trans females) using gonadotrophin-releasing hormone agonists to suppress the endogenous oestrogen and testosterone. ${ }^{[24]}$ The next stage is the use of gender-affirming hormone treatment using oestrogen and testosterone to feminise or masculinise the person's appearance and inducing the desired gender's secondary sex characteristics. ${ }^{[24]}$ It is noteworthy that the effects induced by the hormone medications can be reversed at this stage.

The final stage is gender-affirming surgical procedures for TGD adolescents or adults. These surgical techniques are considered to be irreversible. ${ }^{[24]}$ Chest reconstructive surgery, or "top surgery," is considered an integral part of the transition process. This chest reconstructive surgery can be done for individuals hoping to achieve a more masculine or flat chest, or a more feminine sized and shaped chest. ${ }^{[2]}$ Hormone therapy and living as the person's true gender for 12 months is recommended by the World Professional Association for TG Health ${ }^{[28]}$ before chest surgery and/or surgical castration. Gender re-assignment surgery includes surgical castration, gynaecological procedures including vaginectomy, hysterectomy, and bilateral salpingo-oophorectomy and genital surgery (phalloplasty, scrotoplasty, metoidioplasty). ${ }^{[24]}$

The postoperative acute phase of care of TG patients involves issues with postoperative pain, withdrawal, anxiety and depression in addition to discriminatory health care treatment. ${ }^{[24]}$ Awkward or negative interactions with health care providers may cause psychological discomfort due to the loss of the trusting relationship that should exist between the patient and health care provider. ${ }^{[27]}$ Post surgery participants reported high surgical satisfaction rates despite considerable numbers of postoperative complications, including thrombosis, voiding issues and aesthetics. ${ }^{[29]}$ For trans women the majority of complications were reported after vaginoplasty, mammary augmentation and vocal cord surgery. Trans men had the highest complication rates for penis construction and mastectomy procedures. ${ }^{[29]}$

\section{THE heALTH OF THE TG POPUlation}

As previously mentioned, the overall health outcomes of this population are poorly researched, with the majority of research conducted having focused on the mental health and STIs and HIV status. ${ }^{[25]}$ Acknowledging health care inequalities is only a start to providing much needed support and services to this community. Researchers, educators and all healthcare professional need to have information that is culturally sensitive to best meet the needs of TG people. Some of the issues that may affect access to health care include discrimination, violence, and increased rates of HIV. ${ }^{[3,16]}$

There is evidence to suggest that the majority of TG community delay medical intervention due to discrimination and a lack of understanding, or not wishing to disclose their sexual practices and gender identity. ${ }^{[17]}$ Such delays may result in worse outcomes. Delays in seeking medical treatment occur because of the lack of TG medically trained professionals and could be a causative factor of poor mental health outcomes outlined earlier. An inability to access local general practitioners or councillors they feel comfortable with could be leading to high STI's and HIV within this community, caused by gaps in knowledge of practising safe sex. ${ }^{[30]}$ Sexual practices within this community can lead to higher risks of anal and breast cancer, and with the barriers to medical care/treatment places further delays in timely treatment, leading to poorer outcomes. ${ }^{[31]}$ Furthermore, the TG population has an elevated risk of cardiovascular disease, due to the hormonal treatment required for transition to the opposite sex. ${ }^{[26]}$ This could place them at considerable risk during emergencies when they may not wish to disclose their treatment/gender. ${ }^{[26]}$ The cost of transitioning places a tremendous financial burden on the TG community who already are disadvantaged with employment choices, and homelessness levels, which places them in the lower socioeconomic sector. ${ }^{[21]}$ It has been suggested that WHO should also include gender identity as a social determinant of health, as TG people face many health inequalities which may lead 
to poorer health outcomes. ${ }^{[32]}$ When members of the TG population can express themselves as the gender they wish to identify as, it is known to improve the health and wellbeing of this population. ${ }^{[33]}$ For some members of this population to truly achieve this they require the services of a gender reassignment surgeon. Unfortunately, these services are minimal and come at a high cost to the individual in Australia. ${ }^{[9,10,22]}$ For individuals to be eligible for surgery, they must meet the World Professional Association for Transgender Health Standards of Care guidelines. ${ }^{[34]}$ Those wishing to have surgery must have received psychiatric care for two years and been living at their desired gender for one year to qualify. ${ }^{[4]}$ In Australia, the leading surgeons who can perform gender reassignment surgery, practice in Sydney. ${ }^{[34]}$ This places an additional burden and stress on TG individuals living in other states; increasing costs, removing them from social and family supports, while complicating and increasing costs of continued care. For instance, Australian TG, Cassie Workman, reported these costs to be 85,000 Australian dollars for her to transition to a woman. ${ }^{[35]}$ Currently, approximately 70 reassignment surgeries occur in Australia, but an additional 200 surgeries take place overseas. ${ }^{[34]}$ The Gender Centre in Sydney, Australia, a non-profit organisation that provides services for the TGD community, suggest that the number would fall if more surgeons were performing these surgeries within Australia. This would also reduce the costs with more surgeons creating competition. ${ }^{[34]}$ With the 200 Australians who have their surgery overseas, they are then at higher risk of having complications post-surgery. The reluctance to seek health care and reveal genders could place this 200 at further risk. In addition, they would not only have to face this fear, but they would have to admit to health professionals that they sought treatment outside of Australia, possibly leading to thoughts of guilt or shame. ${ }^{[34]}$ Consequently, they may not seek treatment until complications force them to act. Complications post-surgery can include; bleeding, infections, fistula formation, prolapses, urinary dysfunctions, strictures, implant rejection, implant displacement, necrotising fasciitis, necrosis of the sigmoid conduit and from anywhere between two months and two years, the development of small bowel obstructions. ${ }^{[36,37]}$ This highlights the need for continued post-operative care for the TG population. Post-operative and follow up care, improve surgical and psychosocial outcomes in TG individuals, and the importance of this care needs to be reinforced among the TG community and medical fraternity. ${ }^{[4]}$ Part of the problem in this area, however, is that there is a paucity of evidence base to inform TG clinical care, specifically intervention studies. ${ }^{[38]}$ Unfortunately this contributes to the perpetuation of health inequalities and the invisibility of TG people. ${ }^{[39]}$

\section{Nursing considerations}

Fear of stigma and discrimination from health care providers has been identified by the TG community as an obstacle to seeking healthcare and treatment associated with GDy. ${ }^{[4,34]}$ The Code of Ethics for Nurse in Australia outlines that nurses need to ensure patients are treated with respect regardless of gender or sexual orientation, and providing a nurse/advocate post-operative service will ensure patients are treated with respect and reduce discrimination. ${ }^{[40]}$ The care nurses provide to the TG community must be respectful of their gender identity and value the rights of these individuals to seek gender reassignment surgery. When clients are supported by the nurses post-operatively after receiving the surgery overseas, they will be able to meet the individual needs of these patients within the TG community. Providing a post-operative service to the vulnerable TG community is a way of providing a safe environment. This means than that TG people can share their health concerns without fear of discrimination, fostering a culture that is known to support person-centred care, where a team of supportive health professionals (specialist) assist this community, and they can feel safe to receive treatment. ${ }^{[33,34]}$

To avoid the increased possibility of discrimination, particular care should be taken by health care providers to ensure confidentiality. Furthermore, TG patients should be roomed in hospital according to their gender identity. As stated, TG people generally have higher rates of anxiety and depression than the general population. ${ }^{[23]}$ A multidisciplinary approach incorporating mental health, social work, medical and nursing health care professionals, therefore, should be incorporated over the post-operative period to ease the process of discharge and recovery at home. ${ }^{[23]}$

\section{RECOMMENDATIONS}

In Australia, there is limited capacity within the health system to support the needs of the TGD population. ${ }^{[24]}$ Capacity to improve the primary health needs of this group needs to form collaborative approaches to support services for TG people. Initiatives such as nurse-led post-operative support service need to be created as a way to address this. This service could provide patients with nurse advocates working collaboratively with other health professionals to provide primary health solutions. Nurses can provide post-operative-specific nursing assessments, and liaise with medical professionals, addressing post-operative complications and preventative care. The nurse could then ensure that medical staff have an appropriate background history, removing exposure to any potential bias from the patient, primarily when this is known to be a barrier to accessing care and coordinate relevant appointments formulating an individual care plan. ${ }^{\left[{ }^{34]}\right.}$ Nurses could build positive relationships with supportive health pro- 
fessionals and set up a network of compassionate care for these individuals. These services could be promoted throughout the TG support groups and social media platforms as a centre that supports patients post-operatively regardless of where the surgery occurred, and is known as a safe, supportive environment to promote positive health outcomes in the TG population. This centre could also be promoted within the on-flight media services on flights between Australia and Thailand, for instance. In addition, nurses can provide education to patients on potential post-operative complications, signs and symptoms to look for, and when to seek help, promoting self-care and building capacity in this population. Nurses will also need to develop professional relationships with mental health professionals, social workers, urologists, gynaecologists, endocrinologists, gastroenterologists, plastic surgeons, general practitioners and other specialists when liaising for patients. Through these relationships, the nurses will be gaining extensive knowledge of TG specialist care and educate other professionals with appropriate patient-centred care that is respectful to the TG community through their patient advocate role.

Those who have had gender transformation surgery are able to access care in the state of Victoria. Although this population is small, it is known there are many risks postoperatively, and there is a reluctance and shortage of TG appropriate care for this community, and when the majority of surgeries are conducted in Sydney, patients may forgo aftercare. The problem is that the aftercare received by patients who receive surgery overseas is not to the same standards as in Australia. For instance, patients who received a vaginoplasty in Thailand do not receive a follow-up appointment until one year after surgery. ${ }^{[26,34]}$ In Australia, this would occur at two weeks, placing the Australians who received surgery in Thailand at considerable risk of post-operative complications. ${ }^{[36]}$ The program could utilise some of the 3.4 million Australian dollars allocated for building health capacity of TGD Australians or apply to Victoria Health for funding. The Victorian Government, through Victoria Health, had up to 135,000 Australian dollars available for programs creating social innovations for health equity in 2015. ${ }^{[26]} \mathrm{By}$ providing a respectful person-centred service where there is a known gap, the TG population can feel safe in expressing their concerns, and not feel discriminated against. This will assist in encouraging this population to receive follow up care post-surgery regardless of where they received the surgery.

\section{Providing culturally safe care}

Those who have had gender transformation surgery are able to access care in the state of Victoria. Although this pop- ulation is small, it is known there are many risks postoperatively, and there is a reluctance and shortage of TG appropriate care for this community, and when the majority of surgeries are conducted in Sydney, patients may forgo aftercare. The problem is that the aftercare received by patients who receive surgery overseas is not to the same standards as in Australia. For instance, patients who received a vaginoplasty in Thailand do not receive a follow-up appointment until one year after surgery. ${ }^{[26,34]}$ In Australia, this would occur at two weeks, placing the Australians who received surgery in Thailand at considerable risk of post-operative complications. ${ }^{[36]}$ The program could utilise some of the 3.4 million Australian dollars allocated for building health capacity of TGD Australians or apply to Victoria Health for funding. The Victorian Government, through Victoria Health, had up to 135,000 Australian dollars available for programs creating social innovations for health equity in 2015. ${ }^{[26]} \mathrm{By}$ providing a respectful person-centred service where there is a known gap, the TG population can feel safe in expressing their concerns, and not feel discriminated against. This will assist in encouraging this population to receive follow up care post-surgery regardless of where they received the surgery.

It is important to define what cultural safety mean for TG people. The Nursing Council of New Zealand (2002) has identified a framework that defines cultural safety as incorporating an understanding of how history, culture and power imbalances influence the relationship between the service providers and the population. ${ }^{[17]}$ Cultural safety builds on the health care professionals to have cultural sensitivity and awareness. Education focussing on cultural awareness can provide a shared understanding the needs of TG people. Cultural awareness through education allows health professionals to reflect on their own values and beliefs and the services they provide. ${ }^{[17,41]}$

Cultural awareness and sensitivity of important ethnic and religious diversity incorporating holistic care is planned and delivered in many nursing education and practice settings. ${ }^{[42]}$ However, health professionals practice and education are embedded within a much wider social healthcare system that has failed to address gender outside of birth gender even with a mandate to educate healthcare graduates with competencies in patient centred care. ${ }^{[3,43]}$ In other words, the acknowledgement of cultural awareness regarding people who identify as TG has not fully develop. ${ }^{[38]}$ The reality is that TG culture is invisible. Undergraduate medical and nursing programs need to design and revise their curricula to include TG health as part of a patient-centred model of care that adheres to principles of cultural safety. Clinical placement in community settings that focus on TG people 
could be one way of supporting this improvement. ${ }^{[3,43]}$ It is recommended that a more broad focus on LGBTQ issues needs to be included within undergraduate programs and as part of continued professional development. There is also a need to include more sexuality in health professional curricula which would enable discussion about the issues and care needs of people who identify themselves as TG and help dispel sexual stigma. ${ }^{[44]}$ This would also then help facilitate the conduct of more much needed research in this area and increase the visibility of the TG minority group.

In practice settings, nurses are encouraged to use their clinical judgement in providing patient-centred care for TG people in light of little national data and clinical studies for guidance. ${ }^{[41]}$ However, there is much that clinical nurses can do to improve outcomes in TG clinical interactions through education and communication that reflects dignity and respect. Culturally safe communication uses appropriate language when discussing gender such as asking the patient what they would like to be called, what gender they identify with and what sex they were assigned at birth. ${ }^{[41]}$

\section{Conclusion}

The TG population is a small population within Australia and faces many challenges, health inequalities and inequities. Transgenderism was only recognised as a body dysmorphia and not a psychological disorder in the mid to late 1990's leading to new developments of health services for this population. TG people have significantly poorer mental health outcomes compared to the general population with medically trained professionals being suggested as a causative factor in this due to lack of training and poor sensitivity to the TG population. A lack of engagement with health professionals impacts psychological, physical, social, educational and work life as they are not conforming to birth gender norms.

The health of the TG population can be significantly improved when they can express themselves as the gender they wish to identify as. Some people have sought TG affirming surgery to achieve this. In Australia, there are minimal services that have a high associated cost with a long wait for surgery due to statutory requirements.

Fear of stigma and discrimination from health care providers has been identified by the TG community as an obstacle to seeking healthcare and treatment associated with GDy. Capacity to improve the primary health requirements of this group is needed to form collaborative approaches to support services for TG people. Cultural safety incorporates an awareness through education of the needs of the TG population and the development of sensitivity and empathy. Health professionals challenge their own values and beliefs, in combination with an increased knowledge base, to provide more patient centred care. Undergraduate nursing programs need to design and revise their curricula to include sexuality and TG health as part of a patient-centred model of care that adheres to principles of cultural safety. In addition, there needs to be more research undertaken in this area.

\section{CONFlicts OF INTEREST Disclosure}

The authors declared no conflicts of interest with any of this work, authorship and/or publication.

\section{REFERENCES}

[1] American Psychological Association \& National Association of School Psychologists. Definitions Related to Sexual Orientation and Gender Diversity in APA 2015. Available from: https://www . ap a.org/pi/lgbt/resources/sexuality-definitions.pdf

[2] Wilson T, Shalley F. Estimates of Australia's non-heterosexual population. Australian Population Studies. 2018. Available from: http://www.australianpopulationstudies.org/in dex.php/aps/article/view/23

[3] McPhail D, Rountree-James M, Whetter I. Addressing gaps in physician knowledge regarding transgender health and healthcare through medical education. Canadian Medical Education Journal. 2016; 7(2): e70. https ://doi.org/10.36834/cmej.36785

[4] Coleman E, Bockting W, Botzer M, et al. Standards of Care for the Health of Transsexual, Transgender, and Gender-Nonconforming People, Version 7. International Journal of Transgenderism. 2012; 13(4): 165-232. https://doi.org/10.1080/15532739.2011. 700873

[5] Cooper S, Cant R, Kelly M, et al. An Evidence-Based Checklist for Improving Scoping Review Quality. Clinical Nursing Research. 2019.
PMid:31088144. https ://doi.org/10.1177/10547738198460 24

[6] Morrison SD, Chen ML, Crane CN. Overview of female-to-male gender confirming surgery. Nature Review Urology. 2017; 14: 487-500. PMid:28508877. https ://doi.org/10.1038/nrurol. 2017.64

[7] Kerry SC. Sistergirls/Brotherboys: The Status of Indigenous Transgender Australians. International Journal of Transgenderism. 2014; 15(3-4): 173-186. https ://doi.org/10.1080/15532739. 2014. 995262

[8] Bellhouse C, Walker S, Fairley CK, et al. Patterns of sexual behaviour and sexual healthcare needs among transgender individuals in Melbourne, Australia, 2011-2014. Sexually Transmitted Infections. 2018; 94(3): 212.

[9] Hughes J. Becoming me: How transgender teens navigate the Family Court System. Alternative Law Journal. 2017; 42(4): 261-266. https ://doi .org/10.1177/1037969X17733156

[10] Telfer M, Tollit M, Feldman D. Transformation of health-care and legal systems for the transgender population: The need for change in Australia. Journal of Paediatrics and Child Health. 2015; 
51(11): 1051-1053. PMid:26303157. https://doi.org/10.111 1/jpc.12994

[11] Foreman M, Hove L, York K, et al. Genetic link between gender dysphoria and sex hormone signalling. The Journal of Clinical Endocrinology and Metabolism. 2019; 104(2): 390-396. PMid:30247609. https://doi.org/10.1210/jc.2018-01105

[12] Graves J. How genes and evolution shape gender - and transgender - identity. January 24, 2019. Available from: http://theconversation.com/how-genes-and-evolution - shape-gender-and-transgender-identity-108911

[13] De'Carlo T. Trans History - A Historical Reference Collective of Transgender Events. Morrisville: Lulu. 2018.

[14] Miles-Johnson T. "They don't identify with us". Perceptions of police by Australian transgender people. International Journal of Transgenderism. 2015; 16(3): 169-189. https://doi.org/10.1080/1553 2739.2015 .1080647

[15] Monash Health. Monash Health Gender Clinic. 2019. Available from: https://monashhealth.org/services/services-f-n /gender-clinic/gender-clinic-about-us/

[16] Fernández-Rouco N, Fernández-Fuertes AA, Carcedo RJ, et al. Sexual Violence History and Welfare in Transgender People. Journal of Interpersonal Violence. 2017; 32(19): 2885-2907. PMid:27386885. https://doi.org/10.1177/0886260516657911

[17] Comfort J, McCausland K. Health priorities and perceived health determinants among western Australians attending the 2011 LGBTI Perth pride fairday festival. Health Promotion Journal of Australia. 2013; 24(1): 20-25. PMid:23575585. https ://doi.org/10.107 $1 / \mathrm{HE} 12906$

[18] Bretherton I, Thrower E, Grossmann M, et al. Cross-sex hormone therapy in Australia: the prescription patterns of clinicians experienced in adult transgender healthcare. Internal Medicine Journal. 2019; 49(2): 182. PMid:29992681. https://doi.org/10.1111/ imj. 14035

[19] Mizock L, Mueser KT. Employment, Mental Health, Internalized Stigma, and Coping with Transphobia Among Transgender Individuals. Psychology of Sexual Orientation and Gender Diversity. 2014; 1(2): 146-158. https://doi.org/10.1037/sgd0000029

[20] World Health Organisation. HIV/AIDS. Transgender people (accessed 25/11/2019). Available from: https://www . who.int/hi v/topics/transgender/en/

[21] Oakley S, Bletsas A. The experiences of being a young LGBTIQ and homeless in Australia: Re-thinking policy and practice. Journal of Sociology. 2018; 54(3): 381-395. https://doi .org/10.1177/14 40783317726373

[22] Bell F. Children with gender dysphoria and the jurisdiction of the family court. University of New South Wales Law Journal. 2016; 39(2): 426-454.

[23] Painter M, Palaniappan S. Re: Kelvin - a timing point for gender dysphoria cases in Australia. Law Sopciety of NSW Journal. 2018; 41: 72-3.

[24] Telfer M, Tollit M, Pace C, et al. Australian standards of care and treatment guidelines for transgender and gender diverse children and adolescents. Medical Journal of Australia. 2018; 209(3): 132-136. PMid:29902964. https://doi.org/10.5694/mja17.01044

[25] Guarnero PA, Flaskerud JH. Health and Health Research Needs of the LGBTI Community. Issues in Mental Health Nursing. 2014; 35(9): 721-723. PMid:25162195. https://doi.org/10.3109/016128 40.2013 .879360

[26] Ada SC, Olivia O, Shalem L, et al. Sociodemographic and Clinical Characteristics of Transgender Adults in Australia. Transgender Health. 2019; 3(1): 229-238. PMid:30596151. https://doi.org/ $10.1089 /$ trgh. 2018.0019

Published by Sciedu Press
[27] Tolliche L, Walters C, Radix A, et al. The Perioperative Care of the Transgender Patient. Anesth Analg. 2018; 127(2): 359-366. PMid:29757779. https://doi.org/10.1213/ANE.0000000000 003371

[28] World Professional Association for Transgender Health. Standards of Care for the Health of Transsexual, Transgender, and Gender Nonconforming People. 7th Version (World Professional Association for Transgender Health, 2011)

[29] van de Grift TC, Els Elaut SC, Cerwenka PT, et al. Surgical Satisfaction, Quality of Life, and Their Association After Gender-Affirming Surgery: A Follow-up Study. Journal of Sex \& Marital Therapy. 2018; 44(2): 138-148. PMid:28471328. https://doi.org/10.1 080/0092623X.2017.1326190

[30] Donald C, Ehrenfeld J. The Opportunity for Medical Systems to Reduce Health Disparities Among Lesbian, Gay, Bisexual, Transgender and Intersex Patients. Journal of Medical Systems. 2015; 39(11): 1-7. PMid:26411930. https://doi.org/10.1007/s10916-015 $-0355-7$

[31] Yingling CT, Cotler K, Hughes TL. Building nurses' capacity to address health inequities: incorporating lesbian, gay, bisexual and transgender health content in a family nurse practitioner programme. Journal of Clinical Nursing. 2017; 26(17-18): 2807-2817. PMid:28029727. https://doi.org/10.1111/jocn. 13707

[32] Pega F, Veale JF. The case for the World Health Organization's Commission on Social Determinants of Health to address gender identity. American Journal of Public Health. 2015; 105(3): e58. PMid:25602894. https://doi.org/10.2105/AJPH. 2014.3023 73

[33] Transgender and Intersex Health and Wellbeing Working Group. Transgender and gender diverse health and wellbeing. 2014. Available from: https://www2.health.vic.gov.au/about/popul ations/lgbti-health/trans-gender-diverse

[34] Caminer D. Transgender Surgery. Available from: https ://www.drdavidcaminer. com/transgender-sur gery-dr-caminer-australia-international/2019

[35] Lavoipierre A. The hidden $\$ 100,000$ price tag on being transgender Available from: https://www.abc.net.au/news/2018-03-0 2/the-hidden- \protect $\backslash \mathrm{T} 1 \backslash$ textdollar100, 000-price-t ag-on-being-transgender $/ 9498918$

[36] Thewjitcharoen Y, Srikummoon T, Srivajana N, et al. Hemorrhagic necrosis of small bowel following small bowel obstruction as a late complication of sex reassignment surgery - a gap in transgender care. Journal of Surgical Case Reports. 2018; 11: 1-3. PMid:30443323. https://doi.org/10.1093/jscr/rjy314

[37] Sigurjonsson H, Rinder J, Möllermark C, et al. Male to female gender reassignment surgery: Surgical outcomes of consecutive patients during 14 years. JPRAS Open. 2015; 6(C): 69-73. https: //doi.org/10.1016/j.jpra.2015.09.003

[38] Reisner SL, Deutsch MB, Bhasin S, et al. Advancing methods for US transgender health research. Curr Opin Endocrinol Diabetes Obes. 2016; 23(2): 198-207. PMid:26845331. https://doi .org/10.1 097/MED. 0000000000000229

[39] Coulter RWS, Kenst KS, Bowen DJ, et al. Bisexual funded by the National Institutes of Health of Lesbian, Gay, Bisexual, and Transgender Populations. American Journal of Public Health. 2014 PMid:24328665. https://doi.org/10.2105/AJPH. 2013. 3015 01

[40] Nursing and Midwifery Board of Australia. The ICN Code of Ethics for Nurses. Available from: https: //www.icn.ch/sites/default/files/inline-files/2 012_ICN_Codeofethicsfornurses_\%20ng.pdf 
[41] Neira PM. Caring for the Transgender Patient. Journal of Radiology Nursing. 2017; 36(2): 88-89. https://doi.org/10.1016/j.jr adnu.2017.03.002

[42] Kellett P, Fitton C. Supporting transvisibility and gender diversity in nursing practice and education: embracing cultural safety. Nursing Inquiry. 2017; 24(1): 1-7. PMid:27457725. https ://doi .org/10 $.1111 /$ nin. 12146

[43] Lim F, Johnson M, Eliason M. A National Survey of Faculty Knowl- edge, Experience, and Readiness for Teaching Lesbian, Gay, Bisexual, and Transgender Health in Baccalaureate Nursing Programs. Nursing Education Perspectives. 2015; 36(3): 144-152. https: //doi.org/10.5480/14-1355

[44] Merryfeather L, Bruce A. The invisibility of gender diversity: understanding transgender and transsexuality in nursing literature. Nursing Forum. 2014; 49(2): 110-123. PMid:24387331. https: //doi.org/10.1111/nuf.12061 